\title{
Recent Research and Development on Soil and Foundation Engineering at Railway Technical Research Institute (Japan)
}

\author{
Osamu MURATA \\ General Manager, \\ Structure Technology Div.
}

\begin{abstract}
This paper describes the recent research and development on soil and foundation engineering at the Railway Technical Research Institute, including an outline of the study on the Liquefied Stabilization Soil with wasted fiber materials, barrier walls using cement mixture soil with expanded poly-styrol beads to reduce ground vibration and the system to observe embankment deformation using optical fiber.
\end{abstract}

Keywords : Liquefied Stabilization Soil, barrier walls, optical fiber

\section{Introduction}

The soil and foundation engineering related to railways has been researched and developed by the Foundation \& Geotechincal Engineering Group, Railway Technical Research Institute (R.T.R.I.) in Japan.

As for the earth structure, research has been made on the geosynthetic-reinforced soil retaining wall (GRSRw) to adapt bride abutments for long span bridges. This GRSRw is very stable against strong earthquakes.

Other technologies have been researched and developed including the Liquefied Stabilization Soil( L.S.S.) with wasted fiber materials, cutoff wall using polymer for protection against injurious materials caused by train running and deformation observing system of embankment using optical fiber .

Study that have been made to enhance the technology of the foundation include a system to find damage of foundation, new foundation method and new design method against earthquake.

This paper shows some examples of recent research and development on geotechnical engineering.

\section{Liquefied Stabilization Soil (L.S.S.) with wasted fiber material}

L.S.S. consists of slurry made of on-site soil, water, cement and sand or clay as appropriate L.S.S. is used for backfill at the upper part of a cut and cover tunnel and as an invert material of a shield tunnel (See Fig.1 and Fig.2).

Pit sand is usually used for backfill, but L.S.S. is much better than the sand, because it is easy to use with on-site soil and L.S.S. can be buried without compaction into a narrow space.

The lower part of shield tunnel (see Fig.2) is usually buried by low-strength concrete ( unconfined compressive strength : about $10 \mathrm{MN} / \mathrm{m}^{2}$ ). From the environmental point of view, however, L.S.S., which can reuse onsite soil, is now often used.

Mixture of L.S.S. was designed from the results of unconfined compression tests and repeated loading tests. Then, it was decided that the unconfined compressive

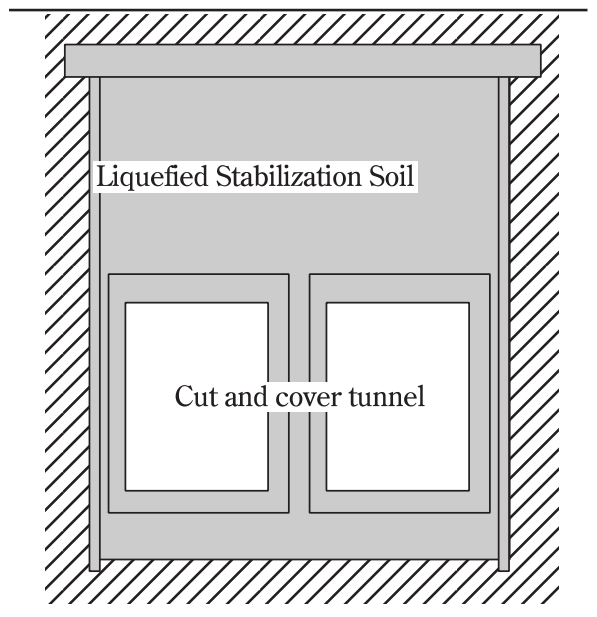

Fig. 1 Liquefied Stabilization Soil used for backfill at upper part of cut and cover tunnel

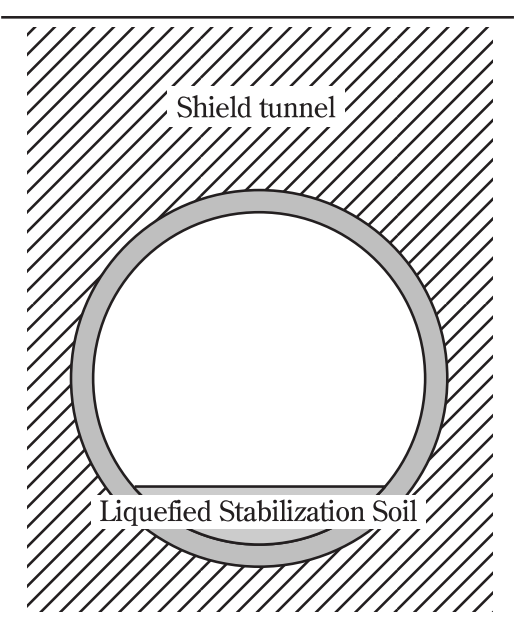

Fig. 2 Liquefied Stabilization Soil used for invert material
of shield tunnel

strength of liquefied soil should be about $6 \mathrm{MN} / \mathrm{m}^{2}$ for safety puropose.

To hold this strength level $\left(6 \mathrm{MN} / \mathrm{m}^{2}\right)$ for some onsite soil, a very large amount of cement is needed ( 300 $\sim 400 \mathrm{~kg}$ weight of cement per $1 \mathrm{~m}^{3}$ of liquefied stabiliza- 
tion soil).

So, the strength level has been reviewed based on repeated loading tests and other tests. A method to mix wasted fiber materials into L.S.S. has been studied in order to increase the unconfined compressive strength and ductility and decrease the total material cost.

Studies have been promoted on what types of wasted fiber material are available and what rigidity level of wasted fiber material is needed.

\section{Barrier walls using cement mixture soil with expanded poly-styrol beads to reduce ground vibration}

Reducing ground vibration caused by train running is one of the most important problems for speed-up.

Lightening the train weight and increasing the track rigidity is one of the means to reduce ground vibration. Barrier walls constructed into the ground are also a countermeasure for that purpose.

As for barrier walls, several types of walls have been proposed. But a practical design method has not yet been proposed to decide the depth, width and rigidity of the barrier wall according to ground conditions and load conditions,.

Then a practical design method will be proposed by numerical analysis, centrifugal simulation and field tests.

From the results of numerical analysis, the rigidity ratio between the ground and barrier wall contribute much to the reduction of ground vibration. The results of analysis also show that it becomes more effective to reduce ground vibration when the barrier with rigidity is lower than the ground rigidity.

Therefore, barrier walls using cement mixture soil with expanded poly-styrol beads is proposed (see Fig.3). The rigidity of wall is much lower than that of the natural ground.

The ground is stirred using an auger first. Then, soil, cement and expanded poly-syrol beads are mixed up as the auger is pulled up gradually. So an improved pile is inserted into the ground. Then, the second pile is inserted similarly and partially overlapping the first pile. This procedure is repeated until the planned wall is completed.

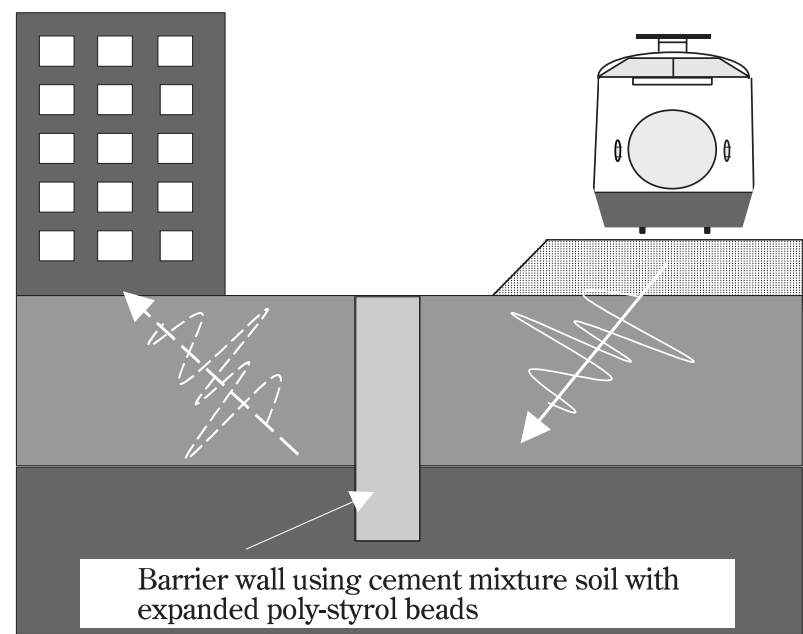

Fig. 3 Barrier wall using cement mixture soil with expanded poly-styrol beads for reducing ground vibration

Execution tests were performed on the site, where the ground is made up from Kanto-loam. Tests were performed for three cases, where volume ratio of expanded poly-styrol beads to the improved pile was $20 \%, 40 \%, 60 \%$. Tests were executed very well without causing any trouble. The modulus of elasticity of the improved piles are about 0.4 $\sim 1.7 \mathrm{MN} / \mathrm{m}^{2}$, while that of the ground is about $3.7 \mathrm{MN} /$ $\mathrm{m}^{2}$. The rigidity of the improved piles is lower than rigidity of ground.

In the future the effectiveness of barrier walls will be measured by this method.

\section{System to observe embankment deformation using optical fiber}

A system to observe embankment deformation using optical fiber has also been studied.

Features of the system using fiber are as follows.

1) Measurement method which can continuously be adopted for long structures.

2) Measurement method which can be adopted for remote sites.

3) Measurement method which can be adopted for a long time.

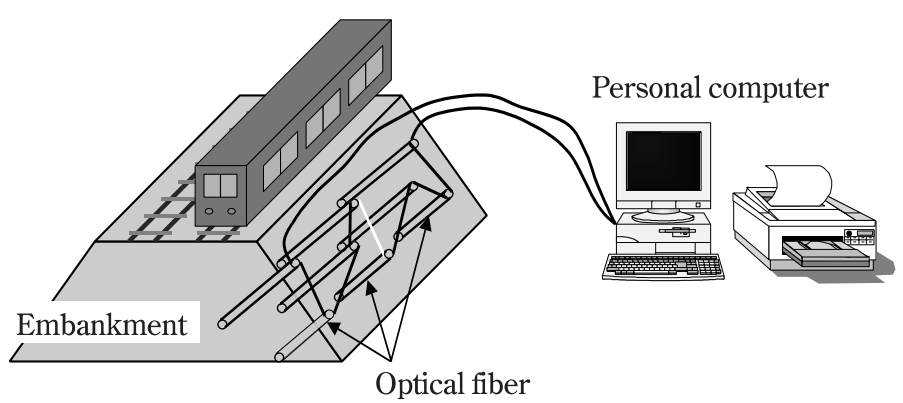

Fig. 4 System to observe embankment deformation using optical fiber 


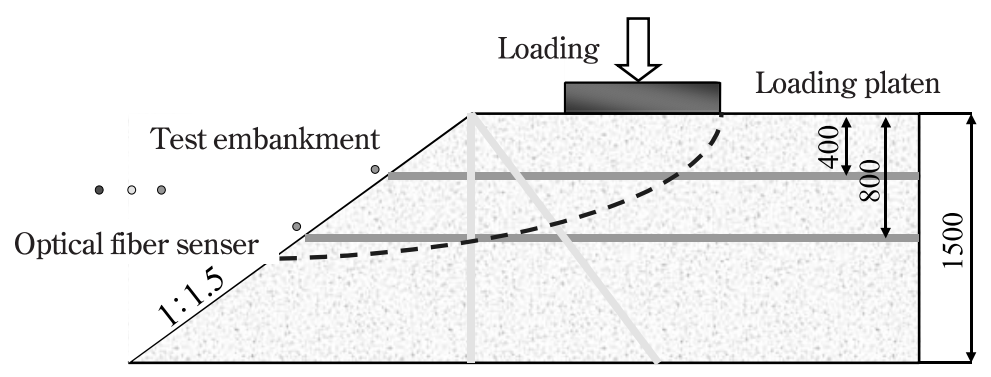

Fig. 5 Loading test using optical fiber

Therefore, this system has been studied for adoption in several fields.

To observe embankment deformation due to heavy rainfall or large earthquake,

a method is proposed to set optical fiber on the surface of embankment and into embankment (see Fig.4).

When this system is adopted for existing embankments, a problem is how to set optical fiber. It is a main research factor to determine how large rigidity of the material attached with optical fiber is reasonable to observe deformation and be inserted into existing embankments.

A loading test of model embankment was performed (see Fig.5). In this test, the embankment was slid intentionally to test several materials attached with optical fiber of different rigidity values for the performance to observe embankment deformation.

Then, a sliding test of model embankment by heavy rainfall was also performed.

It was decided to insert several materials of several rigidity and proper rigidity levels into existing embankments.

From these tests, the system to observe embankment deformation using optical fiber will be completed.

This paper described some examples of recent research and development on the geotechnical field at R.T.R.I.. Besides, new technologies to reduce construction cost and maintenance cost of structures in the geotechnical field have been studied. The results from this study have been used by a number of railway companies. 